\title{
In Silico Analysis of Compounds Isolated from Selected Indian Medicinal Plants against Chikungunya Viral Proteins
}

\begin{abstract}
LAXMI MISHRA, KAVERI TYAGI, MANISHA KUMARI, SHRUTI KHANNA, JUPITA HANDIQUE, MONIKA, NEELAM SACHDEVA, SMRITI SHARMA AND DIVYA GNANESWARI*
\end{abstract}

Department of Zoology, Gargi College, Delhi University, New Delhi-110049

Mishra et al.: Docking of natural compounds with CHIK viral proteins

\begin{abstract}
Chikungunya fever caused by chikungunya virus is an Aedes mosquito borne disease responsible for recent epidemics not only in India but also in Africa, Southeast Asia and America. There are no reports of successful anti-viral drug or vaccine available for the treatment of this fever. Since the symptoms are very severe, development of specific treatment or prophylactic measures are need of the $h$ to control this fever. India is known for its wealth of medicinal plants. Natural compounds from the medicinal plants have been used traditionally for the treatment of several infectious diseases. Indian medicinal plants namely Andrographis paniculata, Tinospora cordifolia and Carica papaya have been selected for this study based on the reports of their usage against this virus in our traditional medicine. The present study evaluates the potentiality of using various compounds derived from these plants as antiviral agent by computationally docking these compounds with some of the viral proteins responsible for its pathogenicity using Auto Dock program. The drug likeness of these compounds was also analyzed based on Lipinski's rule of five. Out of 28 compounds tested, more than 15 compounds are found to be more effective in docking with 5 different viral proteins in comparison with the standard drug prescribed for this fever.
\end{abstract}

Key words: Andrographis paniculata, Tinospora cordifolia, Carica papaya, Chikungunya, docking

Chikungunya is a viral disease transmitted mainly through mosquitoes. It is also reported to be carried by the monkeys, birds and cattle. It was first isolated in Tanzania in $1953^{[1,2]}$. Outbreaks have occurred in many parts of Africa, Southeast Asia Europe and islands in Indian and Pacific oceans. It was first reported in American continent in 2013 to spread throughout the northern, southern and Central America along with Carrabin countries. The rate of death of this disease is 1 out of every $1000^{[2]}$. New born kids, older adults $(>65)$ and patients of high blood pressure, diabetes, heart diseases are at greater risk of being affected. The symptoms include severe joint pains (arthritis) and raised body temperature around $102^{\circ}-104^{\circ} \mathrm{F}$ along with muscular pain, rashes, headache, fatigue, digestive complaints, and conjunctivitis ${ }^{[3]}$.

Chikungunya virus (CHIKV) is a right-handed, singlestranded RNA virus of genome size $11.6 \mathrm{~kb}$. The virus belongs to genus Alphavirus of family Togaviridae ${ }^{[4]}$. Since it is mainly transmitted through arthropodans, it is

*Address for correspondence

E-mail: divyagnaneswari@yahoo.com

July-August 2020 also called arbovirus. The pathogenesis of Chikungunia infection in human is still poorly understood. Till date there is no vaccine for Chikungunya resulting in lack of permanent cure. The patients receive the medical care for the related symptoms which costs even higher than the collective cost of all the symptoms treated as an individual disease. This also increases the burden over the country economy. Research to find out the potential antiviral drug against this re-emerging evolutionary potent CHIK infection is need of the $h$. Indian medicinal plants and its derivatives are widely used in the treatment of several diseases.

Papaya (Carica Papaya) is a tall herbaceous plant growing in tropical regions worldwide. It is native to

This is an open access article distributed under the terms of the Creative Commons Attribution-NonCommercial-ShareAlike 3.0 License, which allows others to remix, tweak, and build upon the work non-commercially, as long as the author is credited and the new creations are licensed under the identical terms 
tropical America and was introduced in India in $16^{\text {th }}$ century. Traditionally the papaya leaves are used for treatment of various ailments such as jaundice, malaria, dengue, immunomodulatory conditions, allergy, digestive complaints and respiratory disorders. It has very strong antiviral activity. The young leaves of Carica are rich in phenolic compounds, (caffeic acid, chlorogenic acid, protocatechuic acid) and flavonoid (kaempferol, quercetin) as compared to mature leaves. Dr. Sanath Hettige reported the efficacy of papaya leaves extract in increasing the White blood cells (WBC) and blood platelets counts in 70 dengue patients. The folic acid in papaya fruit converts homocystenin to cystein and methionine, homosystenin in unconverted state can rupture blood vessel walls resulting in cardiac arrest and stroke. The papaya oil is reported to relieve muscular pain and also helps in overcoming arthritis pain. Root extracts are helpful in urinary infections. The dried leaves of papaya are used as cigar to treat asthma ${ }^{[5,6]}$.

Andrographis paniculata is an annual plant native to Sri Lanka and India. This is a traditional medicinal plant reported to help in cardiovascular diseases. The complete plant is of medicinal importance. The leaves of the plant are rich in lactones (andrographolide, kalmeghin, deoxyandrographolide $)^{[7]}$. Roots are rich in flavonoids which are also found in leaves. The plant extracts has been reported to be hepato-protective, antidiabetic, anti-inflammatory and the treatment with plant extract resulted in dose-dependent fall in systolic blood pressure $^{[8]}$. The potential of andrographolide against CHIKV infection has also been tested in cell culture system $^{[9]}$.

Another medicinally important plant, native to Indian subcontinent and China is Tinospora cordifolia (Giloe) belongs to the family Menispermaceae. The root, leaf, stem, extract of this plant has been established effective in jaundice, diabetes, seminal weakness, urinary tract infections, fever, general debility, skin disease, digestive ailments ${ }^{[10-14]}$, anti-stress, anti-spasmodic, anti-inflammatory ${ }^{[15]}$. The stem is bitter stomachic, diuretic, and also stimulates bile secretion. T. cordifolia extracts are rich in alkaloids, terpenoids, lactone, glycoside, steroid, phenolic compounds and aliphatic compounds etc., which are quite effective to deal with chikungunya symptoms. It has also been studied to be a good immune activator.

These three plants have been widely used for the treatment of chikungunya viral infection in traditional medicine. Based on these reports, the present study was designed to computationally screen the derivatives of these plants through molecular docking against chikungunya viral proteins.

\section{MATERIALS AND METHODS}

\section{Retrieval of CHIK viral proteins:}

The Protein Data Bank (PDB) file of 4 proteins, Macrodomain of CHIK virus (PDB code: 3GPG), immature envelope glycoprotein complex (PDB code: $3 \mathrm{~N} 40$ ), nsP2 protease (PDB code: $4 \mathrm{ZTB}$ ) and cryomicroscopy of Chikungunya protein E1 and E2 (PDB code: 5ANY) were retrieved from the molecular database Repository for biological molecular information (RCBS). All these proteins obtained from RCBS were associated with ligand molecule. PyMOL molecular viewer was used to remove the associated ligands, other hetero atoms and water molecules.

\section{Preparation of protein:}

AutoDock tools were used to prepare the target protein by adding hydrogen atoms at unfulfilled valencies to correct the calculation of partial charges and polar bonds were merged. Gasteiger charges were calculated to each atom of the molecule and total energy calculated. This charged protein was saved as a PDBQT (Protein Data Bank, Partial Charge (Q), \& Atom Type (T)) file.

\section{Ligand retrieval and preparation:}

Based on the literature survey on the compounds isolated from three selected Indian medicinal plants, twelve compounds from $A$. paniculata, seven from C. papaya and nine from T. cordifolia were selected for docking studies. The 3D structures of these compounds were downloaded in SDF (Statutory Declaration Form) format from the PubChem database ${ }^{[16]}$. Avogadro software was used to minimize on energy by optimization of geometry in the force field mmff94.

\section{Docking of the molecule to protein:}

Over the protein molecule, a grid map was created to encompass the active site of the compound using the AutoGrid program. Molecular docking was done using AutoDock 4.2.6, by using genetic algorithm (for semi flexible docking, keeping protein rigid and ligand flexible) ${ }^{[17]}$. Its parameters were set to 10 runs in a population size of 150 , the maximum number of configurations set to 27000 and energetics evaluation to 2500 000. Rest of the parameters was run default. The output was obtained in Lamarckian parameters, and the docking results were saved as a .dlg file, and 
all docked conformations were visualized. The best docking output (in terms of energy and bonding) were selected and exported as a complex. The analysis and visualization of the binding interaction of protein and ligands were performed using PyMol.

\section{Prediction of drug-likeness property:}

The antiviral potential of the selected compounds was predicted using by using the online cheminformatics software Molinspiration ${ }^{[18]}$. According to Lipinski's rule of five, the potential drug should have the following properties (a) less than $500 \mathrm{~g} / \mathrm{mol}$ molecular weight; (b) number of hydrogen-bond donors is less than 3; (c) number of hydrogen-bond acceptors is less than 12; (d) partition coefficient $(\log \mathrm{P})$ is below 5 and (e) no. of rotatable bonds is less than 12 .

\section{RESULTS AND DISCUSSION}

Most of the selected medicinal compounds were found to dock better than the standard drug Acetaminophen prescribed for the treatment of Chikungunya. The best docked ligand molecules were selected based on criterion of lowest binding energy and lesser inhibitory constant (KI). Among the 12 compounds of Andrographis, Andrograpanin showed best result when docked with receptor $3 \mathrm{~N} 40(-7.43 \mathrm{kcal} / \mathrm{mol}$ binding energy and $3.6 \mu \mathrm{M} \mathrm{Ki}$ ) and 14-deoxyadrographolide against 5ANY2 (-6.6 kcal/mol binding energy and $14.48 \mu \mathrm{M} \mathrm{Ki}$ ) (Table 1).

Coumaric acid $(-7.71 \mathrm{kcal} / \mathrm{mol})$ docked well by forming 2 hydrogen bond with 4ZTB.Citrophen of $C$. papaya recorded lowest binding energy of $-5.83 \mathrm{kcal} /$ mol against $3 \mathrm{GPG}$ and $-5.37 \mathrm{kcal} / \mathrm{mol}$ against $3 \mathrm{~N} 40$ and
$-5.17 \mathrm{kcal} / \mathrm{mol}$ against 5ANY2. Quercetin formed three hydrogen bond with 5ANY1 with the lowest binding energy of $-5.16 \mathrm{kcal} / \mathrm{mol}$.

From the compounds isolated from Tinospora cordifolia, Magnoflorine and Berberine found to dock well with 3GPG. They also showed the lowest binding energy against 5ANY1 $(-6.2 \mathrm{kcal} / \mathrm{mol})$ and 4ZTB $(-8.05 \mathrm{kcal} /$ mol). Tinosporide docked with $3 \mathrm{~N} 40$ recorded lowest binding energy $(-6.57 \mathrm{kcal} / \mathrm{mol})$. Magnoflorine by forming one hydrogen bond with 5ANY2 docked well with the binding energy of $-6.57 \mathrm{kcal} / \mathrm{mol}$. Among all the selected compounds of three plants, three compounds of TC (Magnoflorine, Berberine and Tinosporide) docked strongly with most of the viral proteins with lowest binding energy (fig. 1). These compounds also showed excellent drug likeness property based on the Lipinski's rule of five (Table 2). Most of the compounds passed these rules and those violated this rule did not dock well with the selected compounds as well (Table 3-7). In a similar study, Hesperetin was screened for potential inhibitory activities against four CHIKV's nonstructural proteins, namely nsP1, nsP2, nsP3 and nsP4 in addition to SPK2 via the docking of the compound to each protein. Hesperetin exhibited the strongest binding affinity against $3 \mathrm{GPG}(-8.5 \mathrm{kcal} / \mathrm{mol})$, followed by $\mathrm{nsP} 4$ and SPK2 $(-7.7 \mathrm{kcal} / \mathrm{mol}), \mathrm{nsP} 1(-7.6 \mathrm{kcal} /$ $\mathrm{mol})$ and finally 3 TRK $(-6.9 \mathrm{kcal} / \mathrm{mol})^{[19]}$. Similarly, Suramin, an antiparasitic drug, several chemical compounds had been tested for its anti-CHIK activity by docking ${ }^{[20,21]}$. Luteolin and apigenin rich fraction from the ethanolic extract of Cynodon dactylon (L.) (C.dactylon) Perswas evaluated for its anti-Chikungunya potential using Vero cells. The fraction was found to

TABLE 1: DOCKING RESULTS OF TOP THREE COMPOUNDS WITH CHIK PROTEINS

\begin{tabular}{|c|c|c|c|c|c|c|}
\hline $\begin{array}{l}\text { Protein } \\
\text { name }\end{array}$ & Top score & $\begin{array}{l}\text { Plant } \\
\text { name }\end{array}$ & $\begin{array}{l}\text { Binding energy } \\
(\mathrm{Kcal} / \mathrm{mol})\end{array}$ & $\begin{array}{c}\text { Ligand } \\
\text { efficiency }\end{array}$ & $\mathrm{Ki}(\mu \mathrm{M})$ & H-bond \\
\hline & Magnoflorine & $\mathrm{TC}$ & -7.38 & -0.3 & 3.9 & 2 \\
\hline \multirow[t]{3}{*}{$3 G P G$} & Berberine & $\mathrm{TC}$ & -7.34 & -0.29 & 4.17 & 1 \\
\hline & Tinosporide & $\mathrm{TC}$ & -6.24 & -0.23 & 26.61 & 1 \\
\hline & Andrograpanin & AP & -7.43 & -0.32 & 3.6 & 1 \\
\hline \multirow[t]{3}{*}{$3 N 40$} & Tinosporide & $\mathrm{TC}$ & -6.57 & -0.24 & 15.33 & 0 \\
\hline & Berberine & $\mathrm{TC}$ & -6.33 & -0.25 & 22.81 & 0 \\
\hline & Berberine & $\mathrm{TC}$ & -8.05 & -0.32 & 1.26 & 0 \\
\hline \multirow[t]{3}{*}{ 4ZTB } & Coumaric acid & $\mathrm{CP}$ & -7.71 & -0.64 & 2.22 & 2 \\
\hline & Jatorrhizinechloride & $\mathrm{TC}$ & -7.67 & -0.31 & 2.37 & 0 \\
\hline & Magnoflorine & $\mathrm{TC}$ & -6.2 & -0.25 & 28.33 & 0 \\
\hline \multirow[t]{3}{*}{ 5ANYE1 } & Berberine & $\mathrm{TC}$ & -5.78 & -0.23 & 58.13 & 0 \\
\hline & Tinosporide & $\mathrm{TC}$ & -5.76 & -0.21 & 59.46 & 1 \\
\hline & Berberine & TC & -6.61 & -0.26 & 14.23 & 1 \\
\hline \multirow[t]{2}{*}{ 5ANYE2 } & 14-deoxyandrographolide & AP & -6.6 & -0.27 & 14.48 & 0 \\
\hline & Magnoflorine & $\mathrm{TC}$ & -6.57 & -0.26 & 15.26 & 1 \\
\hline
\end{tabular}

$\mathrm{Ki}$ is Inhibitory constant, TC is Tinospora cordifolia , AP is Andrographis paniculata, CP is Carica Papaya 


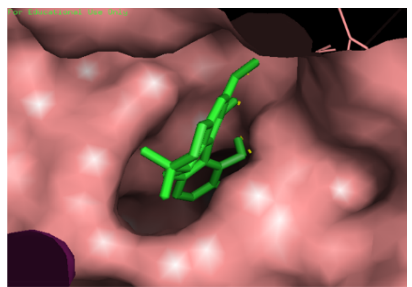

Magn oflorine- 3GPG

Andrograpanin - 3N40

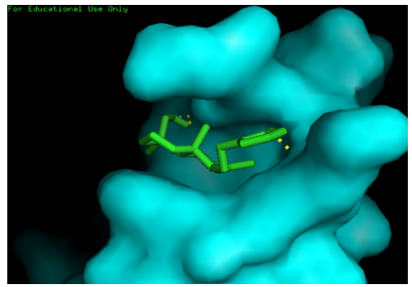

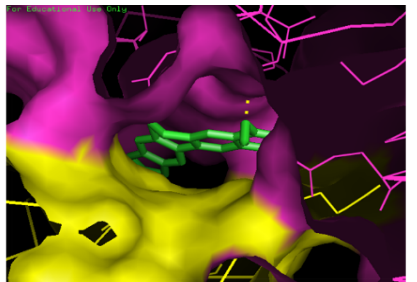

Berberine - 4ZTB

Magnoflorine - 5ANYE 1

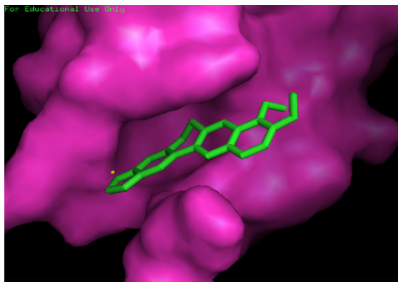

Berberine - 5ANYE2

Acetaminophen - 5ANYE2
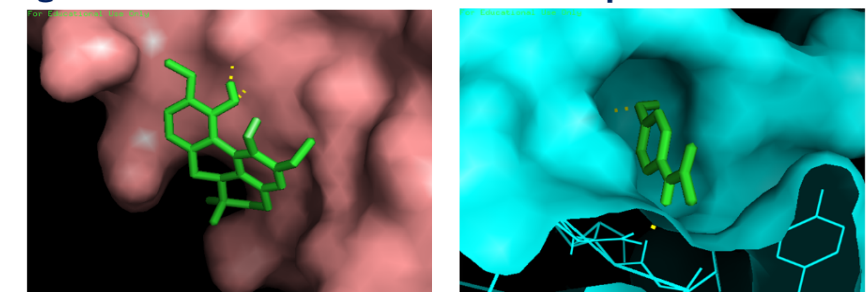

Fig. 1: Binding of top medicinal plant compounds and the standard drug with CHIK proteins

TABLE 2: DRUG-LIKENESS PROPERTY OF THE SELECTED COMPOUNDS OF ALL THE THREE PLANTS BASED ON LIPINSKI'S RULE

\begin{tabular}{|c|c|c|c|c|c|c|c|c|}
\hline S. no. & Pub chem. id & Name of the compound & Mol. Wt (g/mol) & nON & $\mathrm{nOH}$ & $\log P$ & $\mathrm{~N}$ rota bonds & $\mathrm{N}$ viol \\
\hline \multicolumn{9}{|c|}{ Andrographis paniculata } \\
\hline 1. & CID_11624161 & 14-deoxyadrographolide & 334.46 & 4 & 2 & 1.72 & 4 & 0 \\
\hline 2. & CID_73353957 & 14-acetylandrographolide & 392.49 & 6 & 2 & 1.75 & 5 & 0 \\
\hline 3. & CID_11666871 & Andrograpanin & 318.46 & 3 & 1 & 2.87 & 4 & 0 \\
\hline 4. & CID_101652156 & Andrographiside & 512.60 & 10 & 6 & -0.66 & 6 & 2 \\
\hline 5. & CID_5318517 & Andrographolide & 350.45 & 5 & 3 & 1.05 & 3 & 0 \\
\hline 6. & CID_5280443 & Apigenin & 270.24 & 5 & 3 & 2.46 & 1 & 0 \\
\hline 7. & CID_5281780 & 3,4-dicaffeoylquinicacid & 516.46 & 12 & 7 & 1.21 & 9 & 3 \\
\hline 8. & CID_101563021 & Isoandrographolide & 350.45 & 5 & 2 & 1.14 & 2 & 0 \\
\hline 9. & CID_188316 & 7-0-methylwogonin & 298.29 & 5 & 1 & 3.27 & 3 & 0 \\
\hline 10. & CID_9848024 & Neoadrographolide & 480.6 & 8 & 4 & 1.17 & 7 & 0 \\
\hline 11. & CID_42608095 & Onysilin & 300.31 & 5 & 1 & 2.71 & 3 & 0 \\
\hline 12. & CID_101593061 & $\begin{array}{c}\text { 14-deoxy-11- } \\
\text { oxoandrographolide }\end{array}$ & 348.47 & 5 & 2 & 0.62 & 4 & 0 \\
\hline \multicolumn{9}{|c|}{ Carica Papaya } \\
\hline 1. & CID_689043 & Caffeic acid & 180.16 & 4 & 3 & 0.94 & 2 & 0 \\
\hline 2. & CID_1794427 & Chlorogenic acid & 354.31 & 9 & 6 & -0.45 & 5 & 1 \\
\hline 3. & CID_2775 & Citropten & 206.2 & 4 & 0 & 2.03 & 2 & 0 \\
\hline 4. & CID_322 & Coumaric acid & 164.16 & 3 & 2 & 1.43 & 2 & 0 \\
\hline 5. & CID_5280863 & Kaempfeol & 286.24 & 4 & 0 & 2.17 & 1 & 0 \\
\hline 6. & CID_72 & Protocatechuic acid & 154.12 & 4 & 3 & .88 & 1 & 0 \\
\hline 7. & CID_5280343 & Quercetin & 302.24 & 7 & 5 & 1.68 & 1 & 0 \\
\hline \multicolumn{9}{|c|}{ Tinospora cordifolia } \\
\hline 1. & CID_2353 & Berberine & 336.37 & 5 & 0 & 0.20 & 2 & 0 \\
\hline 2. & CID-101676208 & Cordifoliside c & 522.55 & 4 & 2 & 1.14 & 6 & 2 \\
\hline 3. & CID_101915817 & Cordioside & 538.55 & 12 & 5 & 0.19 & 6 & 2 \\
\hline 4. & CID_371256 & Jatorrhizine chloride & 338.38 & 5 & 1 & -0.35 & 3 & 0 \\
\hline 5. & CID_73337 & Magnoflorine & 342.42 & 5 & 2 & -1.26 & 2 & 0 \\
\hline 6. & CID_19009 & Palmatine & 352.41 & 5 & 0 & -0.05 & 4 & 0 \\
\hline 7. & CID_167718 & Tembetarine & 344.43 & 5 & 2 & -1.60 & 4 & 0 \\
\hline 8. & CID_100926540 & Tinocordifolin & 250.34 & 3 & 1 & 2.17 & 1 & 0 \\
\hline 9. & CID_442068 & Tinosporide & 374.39 & 7 & 1 & 2.02 & 1 & 0 \\
\hline \multicolumn{9}{|c|}{ Synthetic drug } \\
\hline 10. & CID_1983 & Acetaminophen & 151.16 & 3 & 2 & 0.68 & 1 & 0 \\
\hline
\end{tabular}

nON is number of hydrogen bond donor, $\mathrm{nOH}$ is number of hydrogen bond acceptor

3GPG is Macrodomain of Chikungunya virus, 3N40 is immature envelope glycoprotein complex, 4ZTB is nsP2 protease and 5ANY is cryomicroscopy of Chikungunya protein E1 and E2. 


\begin{tabular}{|c|c|c|c|c|c|}
\hline$\overline{\text { S.No }}$ & Name of the Compound & Binding Energy (kcal/mol) & Ligand Efficiency & $\overline{\mathrm{Ki}}$ & H-Bond \\
\hline \multicolumn{6}{|c|}{ Andrographis paniculata } \\
\hline 1 & apigenin & -6.18 & -0.31 & $29.36 \mu \mathrm{M}$ & 2 \\
\hline 2 & onysilin & -5.92 & -0.27 & $45.58 \mu \mathrm{M}$ & 1 \\
\hline 3 & methylwogonin & -5.82 & -0.26 & $53.78 \mu \mathrm{M}$ & 1 \\
\hline 4 & andrograpanin & -5.67 & -0.25 & $70.04 \mu \mathrm{M}$ & 1 \\
\hline 5 & isoandrographolide & -5.18 & -0.21 & $160.12 \mu \mathrm{M}$ & 0 \\
\hline 6 & andrographolide & -5.14 & -0.21 & $171.79 \mu M$ & 2 \\
\hline 7 & oxoandrographolide & -5.06 & -0.2 & $194.65 \mu M$ & 2 \\
\hline 8 & andrographiside & -4.61 & -0.13 & $415.18 \mu M$ & 0 \\
\hline 9 & 14-deoxyandrographolide & -4.36 & -0.18 & $639.72 \mu M$ & 0 \\
\hline 10 & acetylandrographolide & -4.21 & -0.15 & $820.31 \mu M$ & 3 \\
\hline 11 & neoandrographolide & -3.37 & -0.1 & $3.38 \mathrm{mM}$ & 2 \\
\hline 12 & dicaffeoylquinic acid & -2.25 & -0.06 & $22.52 \mathrm{mM}$ & 2 \\
\hline \multicolumn{6}{|c|}{ Carica Papaya } \\
\hline 13 & citropten & -5.83 & -0.39 & $53.35 \mu \mathrm{M}$ & 1 \\
\hline 14 & quercetin & -5.69 & -0.26 & $67.72 \mu \mathrm{M}$ & 2 \\
\hline 15 & kaempferol & -5.64 & -0.27 & $73.06 \mu \mathrm{M}$ & 2 \\
\hline 16 & coumaric acid & -5.39 & -0.45 & $111.07 \mu M$ & 2 \\
\hline 17 & caffeeic acid & -4.57 & -0.35 & $450.07 \mu M$ & 1 \\
\hline 18 & chlorogenic acid & -4.26 & -0.17 & $757.67 \mu \mathrm{M}$ & 2 \\
\hline 19 & protocatechuic acid & -4.16 & -0.38 & $885.98 \mu \mathrm{M}$ & 2 \\
\hline \multicolumn{6}{|c|}{ Tinospora cordifolia } \\
\hline 20 & magnoflorine & -7.38 & -0.3 & $3.9 \mu \mathrm{M}$ & 2 \\
\hline 21 & berberine & -7.34 & -0.29 & $4.17 \mu \mathrm{M}$ & 1 \\
\hline 22 & tinosporide & -6.24 & -0.23 & $26.61 \mu \mathrm{M}$ & 1 \\
\hline 23 & tinocordifolin & -5.22 & -0.29 & $149.09 \mu \mathrm{M}$ & 1 \\
\hline 24 & jatorrhizinechloride & -5.12 & -0.2 & $177.99 \mu \mathrm{M}$ & 0 \\
\hline 25 & tembetarine & -5 & -0.2 & $214.92 \mu \mathrm{M}$ & 2 \\
\hline 26 & palmitine & -4.93 & -0.19 & $243.63 \mu \mathrm{M}$ & 1 \\
\hline 27 & cordifoliside C & -3.2 & -0.09 & $4.52 \mathrm{mM}$ & 1 \\
\hline 28 & cordioside & -2.75 & -0.07 & $9.67 \mathrm{mM}$ & 1 \\
\hline \multicolumn{6}{|c|}{ synthetic drug } \\
\hline 29 & acetaminophen & -4.92 & -0.45 & $247.15 \mu M$ & 1 \\
\hline
\end{tabular}

$\mathrm{Ki}$ is Inhibitory constant

TABLE 4: LIST OF LIGANDS AND AUTODOCK BINDING ENERGY, NO. OF HYDROGEN BONDS, INHIBITORY CONSTANT AND LIGAND EFFICIENCY FOR 3N40

\begin{tabular}{|c|c|c|c|c|c|}
\hline S.no & Name of the compound & $\begin{array}{l}\text { Binding energy } \\
(\mathrm{kcal} / \mathrm{mol})\end{array}$ & Ligand efficiency & $\mathbf{K i}$ & $\mathrm{H}$-bond \\
\hline \multicolumn{6}{|c|}{ Andrographis paniculata } \\
\hline 1 & andrograpanin & -7.43 & -0.32 & $3.6 \mu \mathrm{M}$ & 1 \\
\hline 2 & andrographolide & -5.82 & -0.23 & $53.82 \mu \mathrm{M}$ & 2 \\
\hline 3 & isoandrographolide & -5.71 & -0.23 & $65.7 \mu M$ & 1 \\
\hline 4 & methylwogonin & -5.65 & -0.26 & $72.48 \mu \mathrm{M}$ & 0 \\
\hline 5 & 14-deoxyandrographolide & -5.47 & -0.23 & $97.32 \mu \mathrm{M}$ & 1 \\
\hline 6 & onysilin & -5.43 & 0.25 & $104.6 \mu \mathrm{M}$ & 0 \\
\hline 7 & acetylandrographolide & -5.34 & -0.19 & $122.29 \mu \mathrm{M}$ & 1 \\
\hline 8 & apigenin & -5.23 & -0.26 & $147.07 \mu M$ & 1 \\
\hline 9 & oxoandrographolide & -5.12 & -0.2 & $176.92 \mu \mathrm{M}$ & 0 \\
\hline 10 & neoandrographolide & -4.82 & -0.14 & $290.78 \mu \mathrm{M}$ & 1 \\
\hline 11 & andrographiside & -3.89 & -0.11 & $1.4 \mathrm{mM}$ & 0 \\
\hline 12 & dicaffeoylquinic acid & -2.77 & -0.07 & $9.31 \mathrm{mM}$ & 0 \\
\hline \multicolumn{6}{|c|}{ Carica Papaya } \\
\hline 13 & citropten & -5.37 & -0.36 & $115.42 \mu \mathrm{M}$ & 1 \\
\hline
\end{tabular}




\begin{tabular}{|c|c|c|c|c|c|}
\hline 14 & kaempferol & -4.91 & -0.23 & $250.34 \mu \mathrm{M}$ & 0 \\
\hline 15 & quercetin & -4.58 & -0.21 & $438.45 \mu M$ & 0 \\
\hline 16 & coumaric acid & -3.67 & -0.31 & $2.05 \mathrm{mM}$ & 1 \\
\hline 17 & caffeeic acid & -3.63 & -0.28 & $2.17 \mathrm{mM}$ & 3 \\
\hline 18 & protocatechuic acid & -3.26 & -0.3 & $4.07 \mathrm{mM}$ & 1 \\
\hline 19 & chlorogenic acid & -2.62 & -0.1 & $12.06 \mathrm{mM}$ & 2 \\
\hline \multicolumn{6}{|c|}{ Tinospora cordifolia } \\
\hline 20 & tinosporide & -6.57 & -0.24 & $15.33 \mu \mathrm{M}$ & 0 \\
\hline 21 & berberine & -6.33 & -0.25 & $22.81 \mu M$ & 0 \\
\hline 22 & tinocordifolin & -6 & -0.33 & $39.87 \mu M$ & 1 \\
\hline 23 & magnoflorine & -5.77 & -0.23 & $58.8 \mu \mathrm{M}$ & 1 \\
\hline 24 & jatorrhizinechloride & -5.75 & -0.23 & $60.72 \mu \mathrm{M}$ & 0 \\
\hline 25 & palmitine & -5.49 & -0.21 & $94.83 \mu \mathrm{M}$ & 0 \\
\hline 26 & tembetarine & -4.85 & -0.19 & $280.73 \mu \mathrm{M}$ & 1 \\
\hline 27 & cordioside & -3.86 & -0.1 & $1.47 \mathrm{mM}$ & 2 \\
\hline 28 & cordifoliside C & -3.29 & -0.09 & $3.87 \mathrm{mM}$ & 1 \\
\hline \multicolumn{6}{|c|}{ synthetic drug } \\
\hline 29 & acetaminophen & -4.72 & -0.43 & $348.6 \mu \mathrm{M}$ & 1 \\
\hline
\end{tabular}

TABLE 5: LIST OF LIGANDS AND AUTODOCK BINDING ENERGY, NO. OF HYDROGEN BONDS, INHIBITORY CONSTANT AND LIGAND EFFICIENCY FOR 4ZTB

\begin{tabular}{|c|c|c|c|c|c|}
\hline S.no & Name of the compound & Binding energy (kcal/mol) & Ligand efficiency & $\mathrm{Ki}$ & H-bond \\
\hline \multicolumn{6}{|c|}{ Andrographis paniculata } \\
\hline 1 & methylwogonin & -6.69 & -0.3 & $12.39 \mu \mathrm{M}$ & 2 \\
\hline 2 & onysilin & -6.85 & -0.31 & $9.56 \mu \mathrm{M}$ & 0 \\
\hline 3 & apigenin & -6.58 & -0.33 & $14.9 \mu \mathrm{M}$ & 2 \\
\hline 4 & isoandrographolide & -5.96 & -0.24 & $43.02 \mu \mathrm{M}$ & 1 \\
\hline 5 & andrograpanin & -4.8 & -0.21 & $300.59 \mu \mathrm{M}$ & 2 \\
\hline 6 & acetylandrographolide & -4.51 & -0.16 & $491.44 \mu \mathrm{M}$ & 1 \\
\hline 7 & andrographolide & -4.36 & -0.17 & $636.72 \mu \mathrm{M}$ & 0 \\
\hline 8 & 14-deoxyandrographolide & -4.31 & -0.18 & $688.16 \mu \mathrm{M}$ & 1 \\
\hline 9 & neoandrographolide & -4.28 & -0.13 & $722.93 \mu \mathrm{M}$ & 2 \\
\hline 10 & oxoandrographolide & -4.04 & -0.16 & $1.08 \mathrm{mM}$ & 1 \\
\hline 11 & andrographiside & -2.91 & -0.08 & $7.38 \mathrm{mM}$ & 3 \\
\hline 12 & dicaffeoylquinic acid & -2.4 & -0.06 & $17.52 \mathrm{mM}$ & 1 \\
\hline \multicolumn{6}{|c|}{ Carica Papaya } \\
\hline 13 & coumaric acid & -7.71 & -0.64 & $2.22 \mu \mathrm{M}$ & 2 \\
\hline 14 & kaempferol & -6.52 & -0.31 & $16.57 \mu \mathrm{M}$ & 2 \\
\hline 15 & citropten & -6.02 & -0.4 & $38.35 \mu \mathrm{M}$ & 1 \\
\hline 16 & quercetin & -5.84 & -0.27 & $52.25 \mu \mathrm{M}$ & 1 \\
\hline 17 & protocatechuic acid & -5.515 & -0.47 & $166.91 \mu \mathrm{M}$ & 1 \\
\hline 18 & caffeeic acid & -5.5 & -0.42 & $93.49 \mu \mathrm{M}$ & 2 \\
\hline 19 & chlorogenic acid & -5.38 & -0.22 & $114.53 \mu \mathrm{M}$ & 0 \\
\hline \multicolumn{6}{|c|}{ Tinospora cordifolia } \\
\hline 20 & berberine & -8.05 & -0.32 & $1.26 \mu \mathrm{M}$ & 0 \\
\hline 21 & jatorrhizinechloride & -7.67 & -0.31 & $2.37 \mu \mathrm{M}$ & 0 \\
\hline 22 & magnoflorine & -6.69 & -0.27 & $12.38 \mu \mathrm{M}$ & 0 \\
\hline 23 & tinocordifolin & -5.84 & -0.32 & $52.55 \mu \mathrm{M}$ & 0 \\
\hline 24 & tinosporide & -5.21 & -0.19 & $152.19 \mu \mathrm{M}$ & 1 \\
\hline 25 & palmitine & -5.05 & -0.19 & $197.71 \mu \mathrm{M}$ & 0 \\
\hline 26 & cordifoliside C & -3.82 & -0.1 & $1.58 \mathrm{mM}$ & 3 \\
\hline 27 & tembetarine & -2.81 & -0.11 & $8.78 \mathrm{mM}$ & 1 \\
\hline 28 & cordioside & -1.92 & -0.05 & $39.41 \mathrm{mM}$ & 0 \\
\hline \multicolumn{6}{|c|}{ synthetic drug } \\
\hline 29 & acetaminophen & -4.73 & -0.43 & $340.92 \mu \mathrm{M}$ & 2 \\
\hline
\end{tabular}

$\mathrm{Ki}$ is Inhibitory constant 
TABLE 6: LIST OF LIGANDS AND AUTODOCK BINDING ENERGY, NO. OF HYDROGEN BONDS, INHIBITORY CONSTANT AND LIGAND EFFICIENCY FOR 5ANYE1

\begin{tabular}{|c|c|c|c|c|c|}
\hline S.no & Name of the Compound & Binding energy $(\mathrm{kcal} / \mathrm{mol})$ & Ligand efficiency & $\mathrm{Ki}$ & H-bond \\
\hline \multicolumn{6}{|c|}{ Andrographis paniculata } \\
\hline 1 & andrograpanin & -5.68 & -0.23 & $68.41 \mu \mathrm{M}$ & 1 \\
\hline 2 & apigenin & -5.64 & -0.28 & $73.5 \mu \mathrm{M}$ & 3 \\
\hline 3 & onysilin & -5 & -0.23 & $215.66 \mu \mathrm{M}$ & 0 \\
\hline 4 & isoandrographolide & -4.96 & -0.2 & $230.04 \mu \mathrm{M}$ & 2 \\
\hline 5 & methylwogonin & -4.93 & -0.22 & $242.86 \mu M$ & 1 \\
\hline 6 & 14-deoxyandrographolide & -4.59 & -0.19 & $435.14 \mu \mathrm{M}$ & 0 \\
\hline 7 & oxoandrographolide & -4.46 & -0.18 & $534.77 \mu \mathrm{M}$ & 3 \\
\hline 8 & neoandrographolide & -4.2 & -0.12 & $829.34 \mu \mathrm{M}$ & 1 \\
\hline 9 & andrographolide & -4.19 & -0.17 & $848.36 \mu \mathrm{M}$ & 0 \\
\hline 10 & acetylandrographolide & -3.91 & -0.14 & $1.35 \mathrm{mM}$ & 0 \\
\hline 11 & dicaffeoylquinic acid & -2.33 & -0.06 & $19.47 \mathrm{mM}$ & 2 \\
\hline 12 & andrographiside & -1.93 & -0.05 & $38.18 \mathrm{mM}$ & 0 \\
\hline \multicolumn{6}{|c|}{ Carica Papaya } \\
\hline 13 & quercetin & -5.16 & -0.23 & $164.81 \mu \mathrm{M}$ & 3 \\
\hline 14 & kaempferol & -5.15 & -0.25 & $168.35 \mu \mathrm{M}$ & 1 \\
\hline 15 & citropten & -5.01 & -0.33 & $212.47 \mu \mathrm{M}$ & 2 \\
\hline 16 & caffeeic acid & -4.06 & -0.31 & $1.07 \mathrm{mM}$ & 3 \\
\hline 17 & coumaric acid & -3.55 & -0.3 & $2.48 \mathrm{mM}$ & 1 \\
\hline 18 & protocatechuic acid & -3.16 & -0.29 & $4.79 \mathrm{mM}$ & 2 \\
\hline 19 & chlorogenic acid & -2.5 & -0.1 & $14.78 \mathrm{mM}$ & 1 \\
\hline \multicolumn{6}{|c|}{ Tinospora cordifolia } \\
\hline 20 & magnoflorine & -6.2 & -0.25 & $28.33 \mu \mathrm{M}$ & 0 \\
\hline 21 & berberine & -5.78 & -0.23 & $58.13 \mu \mathrm{M}$ & 0 \\
\hline 22 & tinosporide & -5.76 & -0.21 & $59.46 \mu \mathrm{M}$ & 1 \\
\hline 23 & tinocordifolin & -5.72 & -0.32 & $64.57 \mu \mathrm{M}$ & 0 \\
\hline 24 & palmitine & -5.27 & -0.2 & $136.82 \mu \mathrm{M}$ & 0 \\
\hline 25 & tembetarine & -4.97 & -0.2 & $227.57 \mu \mathrm{M}$ & 3 \\
\hline 26 & jatorrhizinechloride & -4.79 & -0.19 & $309.26 \mu \mathrm{M}$ & 0 \\
\hline 27 & cordifoliside C & -3.42 & -0.09 & $3.12 \mathrm{mM}$ & 0 \\
\hline 28 & cordioside & -2.44 & -0.06 & $16.15 \mathrm{mM}$ & 1 \\
\hline \multicolumn{6}{|c|}{ synthetic drug } \\
\hline 29 & acetaminophen & -4.22 & -0.38 & $810.35 \mu \mathrm{M}$ & 2 \\
\hline
\end{tabular}

$\mathrm{Ki}$ is Inhibitory constant

TABLE 7: LIST OF LIGANDS AND AUTODOCK BINDING ENERGY, NO. OF HYDROGEN BONDS, INHIBITORY CONSTANT AND LIGAND EFFICIENCY FOR 5ANYE2

\begin{tabular}{lccccc}
\hline S.no & Name of the compound & Binding energy $\mathbf{( k c a l / m o l )}$ & Ligand efficiency & Ki & H-bond \\
\hline Andrographis paniculata & & & & \\
1 & 14-deoxyandrographolide & -6.6 & -0.27 & $14.48 \mu \mathrm{M}$ & 0 \\
2 & oxoandrographolide & -6.28 & -0.25 & $25.08 \mu \mathrm{M}$ & 2 \\
3 & andrograpanin & -6.09 & -0.26 & $34.57 \mu \mathrm{M}$ & 0 \\
4 & andrographolide & -5.99 & -0.24 & $40.85 \mu \mathrm{M}$ & 0 \\
5 & methylwogonin & -5.94 & -0.27 & $44 \mu \mathrm{M}$ & 0 \\
6 & onysilin & -5.62 & -0.26 & $75.72 \mu \mathrm{M}$ & 0 \\
7 & apigenin & -5.47 & -0.27 & $97.83 \mu \mathrm{M}$ & 0 \\
8 & neoandrographolide & -5.46 & -0.16 & $99.94 \mu \mathrm{M}$ & 2 \\
9 & isoandrographolide & -5.42 & -0.2 & $105.7 \mu \mathrm{M}$ & 0 \\
10 & acetylandrographolide & -5.02 & -0.18 & $209.8 \mu \mathrm{M}$ & 0 \\
11 & dicaffeoylquinic acid & -4.05 & -0.11 & $1.07 \mathrm{mM}$ & 1 \\
12 & andrographiside & -3.47 & -0.1 & $2.87 \mathrm{mM}$ & 3 \\
Carica Papaya & & & & \\
13 & kaempferol & -5.22 & -0.25 & $149.62 \mu \mathrm{M}$ & 0 \\
14 & citropten & -5.17 & -0.34 & $163.2 \mu \mathrm{M}$ & 0
\end{tabular}


www.ijpsonline.com

\begin{tabular}{|c|c|c|c|c|c|}
\hline 15 & caffeeic acid & -4.68 & -0.36 & $373.45 \mu \mathrm{M}$ & 3 \\
\hline 16 & coumaric acid & -4.53 & -0.38 & $474.86 \mu \mathrm{M}$ & 3 \\
\hline 17 & protocatechuic acid & -4.51 & -0.41 & $494.49 \mu \mathrm{M}$ & 3 \\
\hline 18 & quercetin & -4.5 & -0.2 & $502.6 \mu \mathrm{M}$ & 2 \\
\hline 19 & chlorogenic acid & -3.84 & -0.15 & $1.53 \mathrm{mM}$ & 1 \\
\hline \multicolumn{6}{|c|}{ Tinospora cordifolia } \\
\hline 20 & berberine & -6.61 & -0.26 & $14.23 \mu \mathrm{M}$ & 1 \\
\hline 21 & magnoflorine & -6.57 & -0.26 & $15.26 \mu \mathrm{M}$ & 1 \\
\hline 22 & tinosporide & -6.14 & -0.23 & $31.52 \mu \mathrm{M}$ & 0 \\
\hline 23 & palmitine & -6.11 & -0.24 & $33.33 \mu \mathrm{M}$ & 1 \\
\hline 24 & jatorrhizinechloride & -5.83 & -0.23 & $53.51 \mu \mathrm{M}$ & 0 \\
\hline 25 & tinocordifolin & -5.39 & -0.3 & $112.63 \mu \mathrm{M}$ & 1 \\
\hline 26 & tembetarine & -4.91 & -0.2 & $252.12 \mu \mathrm{M}$ & 0 \\
\hline 27 & cordifoliside C & -3.51 & -0.09 & $2.69 \mathrm{mM}$ & 2 \\
\hline 28 & cordioside & -3.24 & -0.09 & $4.2 \mathrm{mM}$ & 3 \\
\hline \multicolumn{6}{|c|}{ synthetic drug } \\
\hline 29 & acetaminophen & -4.85 & -0.44 & $277.96 \mu \mathrm{M}$ & 2 \\
\hline
\end{tabular}

$\mathrm{Ki}$ is Inhibitory constant

exhibit potent viral inhibitory activity (about $98 \%$ ) at the concentration of $50 \mu \mathrm{g} / \mathrm{mL}$ as observed by reduction in cytopathic effect. The cytotoxic concentration of the fraction was calculated and found to be $250 \mu \mathrm{g} / \mathrm{mL}^{[22]}$. The present study shows, Tinospora cordifolia as the most promising anti-viral candidate for Chikungunya. Detailed in vitro/in vivo and pharmaceutical analysis is required to confirm and establish the potentiality of compounds from $T$. cordifolia in controlling the menace caused by Chikungunya virus. Successful compounds short listed from other two plants could also be further investigated for the control of this disease. The lowest binding energy was observed against NSP2 protease which suggests that it can be used as one of the potential drug target. The nsP2 protease was responsible for cleavage in the non-structural polyprotein that are crucial for the viral replication cycle. The inhibitor blocks the protease when new viral particle break off from on infected cells ${ }^{[23]}$.

\section{Acknowledgment:}

We are thankful to the Department of Biotechnology - Star College Scheme for providing the funds and facilities.

\section{REFERENCES}

1. Ross RW. A laboratory technique for studying the insect transmission of animal viruses, employing a bat-wing membrane, demonstrated with two African viruses. Epidemiol Infect 1956;54:192-200.

2. Robinson MC. An epidemic of virus disease in Southern Province, Tanganyika Territory, in 1952-53; Trans R Soc Trop Med Hyg 1955;49:28-32.

3. Caglioti C, Lalle E, Castilletti C, Carletti F, Capobianchi MR,
Bordi, L. Chikungunya virus infection: an overview. New microbiol 2013;36:211-27.

4. Powers AM, Logue $\mathrm{CH}$. Changing patterns of Chikungunya virus: re-emergence of a zoonotic arbovirus. J Gen Virol 2007;88:2363-77.

5. Powers AM, Brault AC, Shirako Y, Strauss EG, Kang W, Strauss $\mathrm{JH}$, et al. Evolutionary relationship and systematics of the alphavirus. J Virol 2001;75:10118-31.

6. Arvind G, Bhowmik D, Duraivel S, Harish G. Traditional and medicinal uses of Carica papaya. J Med Plt Stu 2013;1:7-15.

7. Weiming C, Xiaotian L. Deoxyandrographolide- 19ß-Dglucoside from the leaves of Andrographis paniculata. Planta Med 1982;45:245-6.

8. Zhang XF, Tan BK. Antidiabetic property of ethanolic extract of Andrographis paniculata in streptozotocin-diabetic rats. Acta Pharmacol Sin 2000;21:1157-64.

9. Nadkarni K, Nadkarni AK. Indian Materia Medica, Popular Prakashan Pvt. Ltd., Bombay. 1976;1:799.

10. Kirtikar KR, Basu BD. Indian Medicinal Plants. New Connaught Place, Dehra Dun: M/S Bishen Singh, Mahendra Pal Singh; 1975.

11. Chopra RN, Nayar SL, Chopra IC, Asolkar L, Kakkar K. Glossary of Indian medicinal plants, New Delhi. CSIR 1956.

12. Chopra RN, Chopra LC, Handa KD, Kapur LD, editors. Indigenous Drugs of India. 2nd ed. Kolkata: M/S Dhar VN \& Sons; 1982.

13. Zhao TF, Wang X, Rimando AM, Che CT. Folkloric medicinal plants: Tinospora sagittata var. cravaniana and Mahonia bealei. Planta Med 1991;57:505.

14. Rai M, Gupta SS. The deposition of the secondary salts over the five pellets in rats was inhibited by the aqueous extract of T. cordifolia. J Res Ind Med 1966;10:113-6.

15. Pendse VK, Dadhich AP, Mathur PN, Bal MS, Madan BR. Anti-inflammatory, immunosuppressive and some related pharmacological actions of the water extract of Neem Giloe (Tinospora cordifolia): A preliminary report. Indian J Pharmacol 1977;9:221-4.

16. (https://pubchem.ncbi.nlm.nih.gov/)

17. Morris GM, Huey R, Lindstrom W, Sanner MF, Belew 
RK, Goodsell DS, et al. AutoDock4 and AutoDockTools4: automated docking with selective receptor flexibility. J Comp Chem 2009;30:2785-91.

18. (http://www.molinspiration.com/).

19. Oo A, Hassandarvish P, Chin SP, Lee VS, Bakar SA, Zandi $\mathrm{K}$. In silico study on anti-Chikungunya virus activity of hesperetin. PeerJ. 2016;4:e2602.

20. Ho YJ, Wang YM, Lu JW, Wu TY, Lin LI, Kuo SC, et al. Suramin inhibits chikungunya virus entry and transmission. PloS one 2015;10:e133511.
21. Das PK, Puusepp L, Varghese FS, Utt A, Ahola T, Kananovich DG, et al. Design and Validation of Novel Chikungunya Virus Protease Inhibitors. Antimicrob Agents Chemother 2016;60:7382-95.

22. Murali KS, Sivasubramanian S, Vincent S, Murugan SB, Giridaran B, Dinesh S, et al. Anti-chikungunya activity of luteolin and apigenin rich fraction from Cynodon dactylon. Asian Pac J Trop Med 2015;8:352-8.

23. Bora L. Homology modeling and docking to potential novel inhibitor for chikungunya (37 997) protein nsp2 protease. J Proteomics Bioinform 2012;5:54-9. 